\title{
Young adult mortality in Israel during the COVID-19 crisis
}

\author{
Steve Ohana, Alexandra Henrion Caude ${ }^{1}$ \\ 1 French Institute of Health and Medical Research
}

Funding: The author(s) received no specific funding for this work.

Potential competing interests: The author(s) declared that no potential competing interests exist.

\begin{abstract}
In this paper, we analyze excess mortality in Israel during the COVID-19 crisis, focusing on the age group of young adults under 50 years of age, as their susceptibility to COVID-19 mortality is low. Based primarily on online data from the Central Bureau of Statistics of Israel, we observed an unexpected rise of excess mortality among 20 to 49-year-olds in February-March 2021. It should be noted that excess mortality peaks among these young age groups are rarely observed, with low number of deaths that are usually caused by wars. We examined whether COVID-19 could account for this excess mortality. The inconsistency between the reported COVID-19 deaths and the excess deaths within this age group led to consider other potential causes: accident and vaccination. Indeed, the surge in mortality coincided with the rollout of the Israeli vaccination campaign for the 20 sto 49 -year-olds, which reached more than $75 \%$ of individuals in this age group. This unexpected rise in excess mortality among young adults was also found in two other countries, the United Kingdom and Hungary, which have in common with Israel a massive vaccination of their populations. Thus, our observations should prompt to pause the campaign, while clarifying the underlying reasons for those excess deaths, especially in the context of a low mortality risk from COVID-19 within adults under 50 years of age.
\end{abstract}

\section{Introduction}

Widely-cited studies have concluded to the efficiency of the Pfizer Comirnaty injections to prevent COVID19 infections, hospitalizations and deaths in Israel (see [1] and [2]). Excess mortality by ethnic group, city and age in Israel from March to November 2020 was previously reported [3]. However, to the best of our knowledge, no study analyzed the mortality in the young adult population in Israel after the start of the Israeli vaccination campaign in December 2020.

Overall mortality data (both COVID and non-COVID related) have several advantages over COVID-19 mortality data for analyzing the impact of vaccination, since (i) they are less susceptible to bias or manipulation than "COVID-19 deaths," which may vary in definition and practical calculation depending on the context, and (ii) they capture possible "collateral effects" of vaccination beyond COVID-19 deaths, such as its possible impact on youth mortality. The goal of this paper is to analyze excess mortality in 
Israel in 2020-2021, focusing on those adult age groups that are less susceptible to COVID19 mortality: the group of the 20 to 49 -year-olds.

\section{Results}

\section{Status of excess mortality in 20-49 age groups}

We focused on the mortality of adults under the age of 50 in Israel in 2020-2021 for it is a subject of limited documentation. Focusing on the 20-49 age group limits the confounding effect of COVID-19 mortality and avoids the bias due to the "harvesting" effect of premature deaths in 2020 of individuals with poor health condition, whose health status deteriorated because of both lockdowns and COVID-19. Of note, mortality is seasonal in nature, hence should be adjusted for systematic patterns within the year. Moreover, mortality must be related to the population in order to be compared from year to year (when the population increases, the number of deaths also increases). The number of deaths relative to the population defines the population-adjusted "mortality rate". In some age groups, the mortality rate shows a trend over time, which may reflect -for example- medical advances and improvements in the health status of the population. Excess mortality is therefore studied as the deviation of the mortality rate from that expected from long-term drift and seasonal patterns.

Excess mortality in Israel was calculated on a monthly basis, as shown in Figure 1. The excess mortality was assessed as the difference between the monthly observed mortality rate and the expected mortality rate calculated from a linear model including a long-term linear drift and monthly seasonal dummies. Unusual events of national interest (including wars) were reported. The deaths that occurred on the day of a dramatic accident, which took place in Meron on April 30, 2021, were carefully taken into consideration to avoid any bias, and data was plotted with and without this event. Because the cause is accidental, deaths from the Meron tragedy were systematically discarded from the rest of this paper.

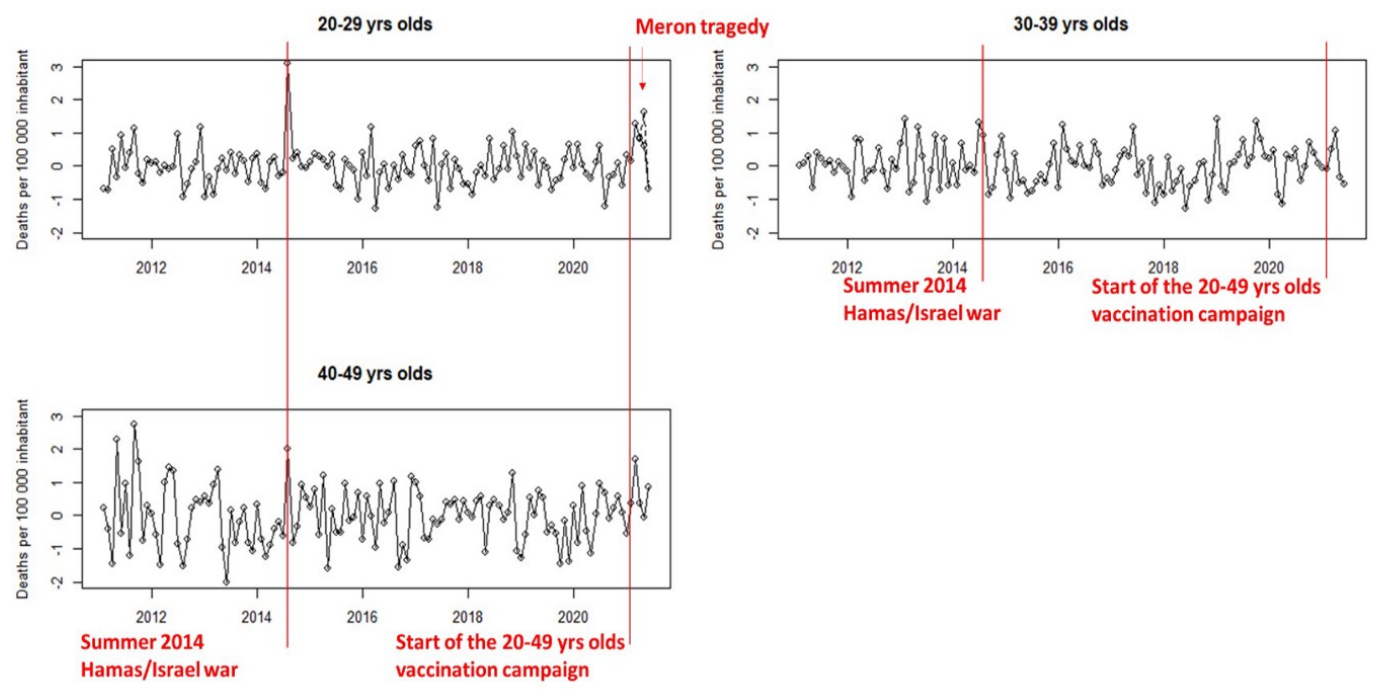

Figure 1: Monthly excess mortality rates in Israel from January 2011 to May 2021. Mortality rates are expressed in number of monthly deaths per 100,000 inhabitants. A graph was plotted per each age group 
20-29, 30-39 and 40-49-year-olds. To ensure data consistency despite uneven number of days in each month, the monthly deaths were converted in deaths per 30 days. Annual population in each age tranche was linearly interpolated to obtain smoothed monthly population data. The excess mortality rate is defined as the mortality rate exceeding a long-term drift and seasonal effects, calibrated by linear model (by age) with a linear trend and monthly dummy variables over the period 2011-2021. For the 20-29-year-olds only, data are indicated with (dashed line) and without (plain line) the Meron tragedy (April 30, 2021), where 45 people (including 15 between 20 and 29 and 16 below 19) lost their lives. Red lines indicate the main unusual national events: in July 2014, the ground offensive of the Operation Protective Edge and in January 2021 , the start of the vaccination campaign for these age categories.

Compared to previous mortality peaks, the peaks between February to April 2021 occurred in all three age groups and lasted two months in a row in the 20-39 age groups. This led us to aggregate excess mortality rates with respect to both the time and age dimensions (Figure 2).

We plotted the three-month moving average of the 20 to 49 -year-olds excess mortality rates, as shown in the lower panel of Figure 2. Intriguingly in 2021, the values in March (average of the January-February and March excess mortality rates) and in April (average of the February-March-April excess mortality rates) exhibited the same magnitude as the peak during the Israeli ground offensive in Gaza in July 2014, as part of Operation Protective Edge, where 67 Israeli soldiers lost their lives.

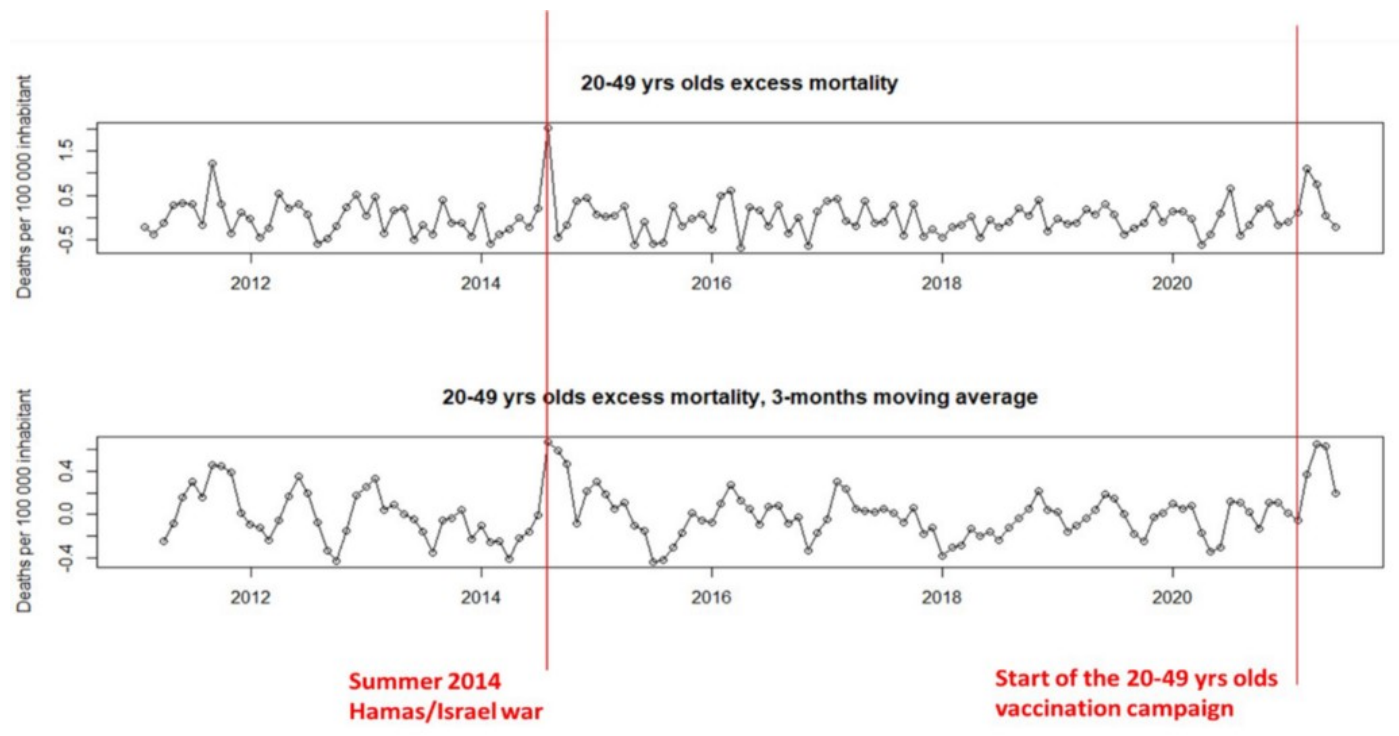

Figure 2: Upper panel: Mortality rate in the age group of the 20-49-year-olds (weighted average of the 2029, 30-39 and 40-49-year-olds mortality rates). The excess mortality rates are defined as the mortality rates exceeding a long-term drift and seasonal effects, calibrated by a linear model with a linear trend and monthly dummy variables. All data are shown without the deaths from the Meron tragedy (April 30, 2021). Red lines indicate the main unusual national events: in July 2014, the ground offensive of the Operation Protective Edge and in January 2021, the start of the vaccination campaign for these age categories. Lower panel: Graph was obtained from a three-month moving average on the time series plotted in the upper 
graph.

\section{Assessing the potential causes of excess mortality}

We focused on COVID-19 as a potential underlying cause of such excess mortality. In Figure 3, we compiled the official COVID deaths with the excess deaths, assessed by the above excess mortality rates both in the age groups below and above 50 years old. As expected, the rise in the excess mortality was parallel to the rise of COVID-19 deaths both in October 2020 and in January 2021. However, an inconsistency arises between the official COVID-19 deaths and excess deaths below 50 years of age starting in February 2021.

Moreover, Figure 3 casts new lights on unreported rises in the young adult mortality that cannot be explained by the overall trend of COVID-19 deaths. While three mortality peaks occur respectively in June 2020, October 2020, and in February-March 2021, only the one in October 2020 coincides with an increase in COVID-19 deaths. Since this peak corresponds to the lowest rise in deaths, and the two other increases cannot be explained by the overall trend of COVID-19 deaths, the need to understand the reason for the last peak (the one with the highest magnitude) appears all the more critical. The only unprecedented noticeable event in this timeframe was the beginning of the vaccination campaign in Israel, which took place in January 2021 for this age group. We thus calculated the proportion of vaccinated young adults as documented in Table 1 from January to May 2021, and observed that February and March 2021 happen to be the two months with the highest number of second doses administered among the injection months (28\% of the 20-49 age group having received their second dose in each of these two months). Thus, from the data put altogether, the only event that can plausibly account for the repeated highest rises in excess deaths both in February and March 2021 is the vaccination.

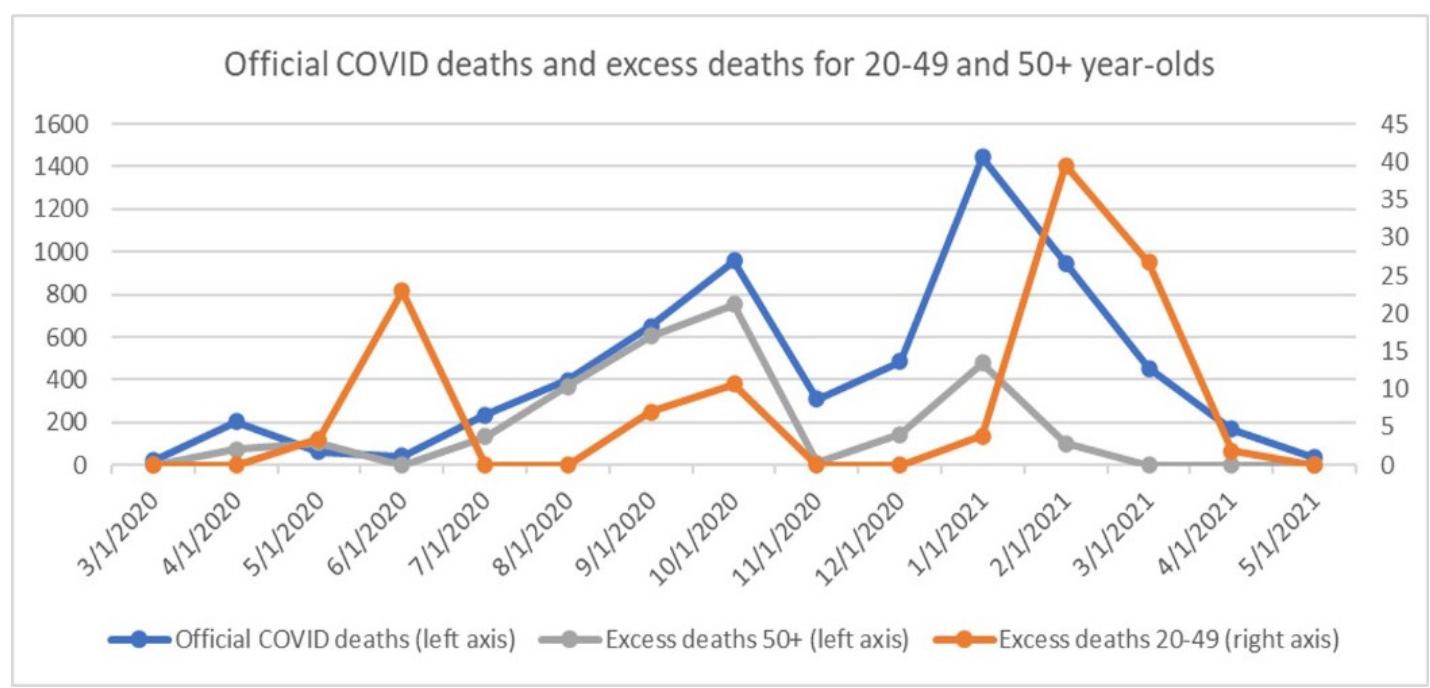

Figure 3: Monthly official (all ages) COVID deaths (left axis) and excess deaths for the 20-49-year-olds (right axis) and the above 50-year-olds (left axis). The excess deaths are computed from the excess mortality rates presented above (retaining only positive values). Source for official COVID deaths: Israeli 
Ministry of Health

\begin{tabular}{|r|r|r|r|r|}
\hline Jan-21 & Feb-21 & Mar-21 & Apr-21 & May-21 \\
\hline $13 \%$ & $41 \%$ & $69 \%$ & $74 \%$ & $76 \%$ \\
\hline
\end{tabular}

Table 1: Proportion of fully vaccinated among the 20 to 49 -year-olds at the end of each month. Source: Israeli Ministry of Health

\section{Comparison with other countries}

We chose to compare the mortality growth of young adult population in countries with varying policies of vaccination. As a comparison, we compiled the mortality rate variation from Jan-May 2020 to Jan-May 2021 in the various age groups in 25 European countries as well as in Israel, as shown in the Appendix in Table A1. The average mortality growth in percentage for each age group was further calculated in mass and moderate vaccination countries (Appendix, Table A2). The p-value of a one-sided (equal variance) t-test was assessed and showed that the two averages were significantly different (Table A2). This table was subsequently presented as a histogram (Figure 4). From this figure and our calculation, it appears that the difference between mass and moderate vaccination is significant in terms of mortality growth in young age groups, and that mass vaccination is associated to a higher young adults' mortality growth. This observation lends further support to suggest a link between vaccination and youth mortality in Israel, and in those countries that perform mass vaccination.

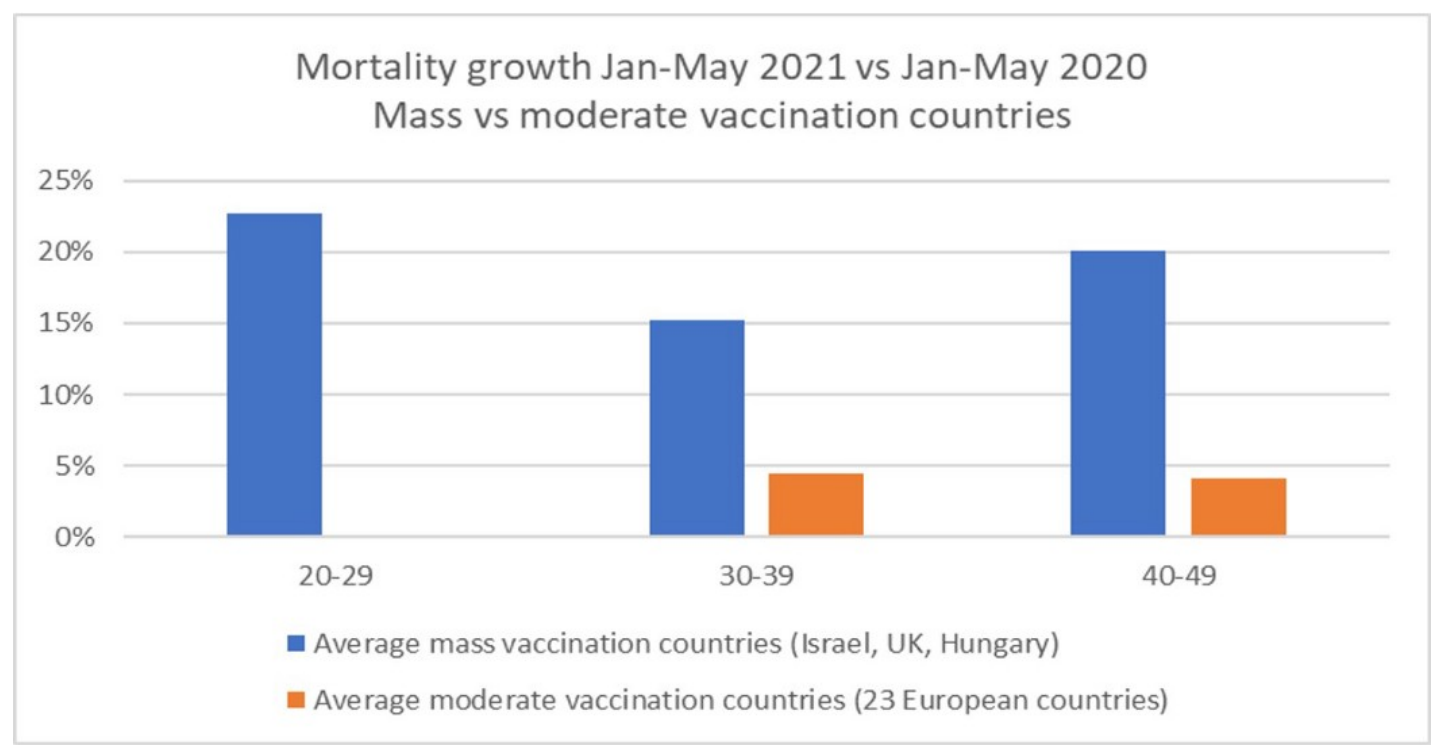

Figure 4: Mortality growth in \% from Jan-May 2020 to Jan-May 2021 in Israel, England \& Wales, Hungary and 23 other European countries which have more moderately vaccinated their populations, for ages 20-

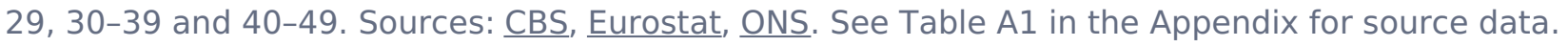




\section{Discussion}

Surges in mortality among young people are very rare events, and are usually associated with wars, such as the peak of excess mortality during the Hamas/Israel war in July 2014. The mortality peak among 20 to 49-year-olds in February-March 2021 is therefore unprecedented and indeed concerning. After eliminating accidents, such as the Meron tragedy, the unusual event which took place within this timeframe was the fact that a very high percentage of Israelis aged of 20-49 years received two doses of Comirnaty mRNA injection.

Our additional observation supporting this possible link between vaccination and youth mortality is the fact that common patterns of excess mortality were also observed in Hungary and England \& Wales, which have in common with Israel a massive vaccination of their populations. The recent research by Retsef Levi [4] documenting a 20 to 35\% increase in cardiac arrests among the Israelis aged 16-49 from Jan-May 2019-2020 to Jan-May 2021 and the established link between the Pfizer vaccine and myocarditis [5]/autoimmune diseases [6] in young adults further lend a physiological support. Such accumulation of concern should, in our view, urgently prompt a pause in the vaccination campaign, until the reasons of the youth excess mortality observed in mass vaccination countries are clarified.

\section{References}

[1] Haas, Eric J., et al. "Impact and effectiveness of mRNA BNT162b2 vaccine against SARS-CoV-2 infections and COVID19 cases, hospitalizations, and deaths following a nationwide vaccination campaign in Israel: an observational study using national surveillance data." The Lancet 397.10287 (2021): 1819-1829. [2] Dagan, Noa, et al. "BNT162b2 mRNA COVID19 vaccine in a nationwide mass vaccination setting." New England Journal of Medicine 384.15 (2021): 1412-1423.

[3] Haklai, Ziona, et al. "Excess mortality during the COVID-19 pandemic in Israel, March-November 2020: when, where, and for whom?." Israel Journal of Health Policy Research 10.1 (2021): 1-7.

[4] See this tweeter thread by Ran Israel on Retsef Levi's research

and Retsef Levi's youtube video explaining his research:

[5] Vogel G., Couzin-Frankel, G., Israel reports link between rare cases of heart inflammation and COVID19 vaccination in young men, Science Mag, June 1, 2021

[6] Jaffe-Hoffman, M., Pfizer COVID19 vaccine linked to rare blood disease - Israeli study, Jerusalem Post, June 24, 2021

\section{Appendix}




\begin{tabular}{|c|c|c|c|c|c|}
\hline & Country \ Age group & $20-29$ & 30-39 & $40-49$ & fully vaccinated per hundred $(31 / 05 / 2021)$ \\
\hline \multirow{3}{*}{$\begin{array}{l}\text { Mass vaccination } \\
\text { countries }\end{array}$} & Israel & $12 \%$ & $6 \%$ & $7 \%$ & 59 \\
\hline & United Kingdom & $13 \%$ & $9 \%$ & $8 \%$ & 38 \\
\hline & Hungary & $43 \%$ & $30 \%$ & $45 \%$ & 38 \\
\hline \multirow{23}{*}{$\begin{array}{c}\text { Moderate vaccination } \\
\text { countries }\end{array}$} & Cyprus & $36 \%$ & $-6 \%$ & $7 \%$ & 24 \\
\hline & Lithuania & $-3 \%$ & $14 \%$ & $-4 \%$ & 22 \\
\hline & Denmark & $-5 \%$ & $5 \%$ & $-10 \%$ & 22 \\
\hline & Switzerland & $-13 \%$ & $-19 \%$ & $-10 \%$ & 21 \\
\hline & Spain & $-10 \%$ & $-1 \%$ & $-2 \%$ & 20 \\
\hline & Slovenia & $-38 \%$ & $4 \%$ & $-7 \%$ & 20 \\
\hline & Greece & $2 \%$ & $9 \%$ & $10 \%$ & 20 \\
\hline & Portugal & $-25 \%$ & $16 \%$ & $2 \%$ & 19 \\
\hline & Romania & $-2 \%$ & $10 \%$ & $16 \%$ & 19 \\
\hline & Belgium & $8 \%$ & $-3 \%$ & $0 \%$ & 19 \\
\hline & Norway & $-8 \%$ & $-16 \%$ & $-19 \%$ & 19 \\
\hline & Poland & $14 \%$ & $29 \%$ & $36 \%$ & 19 \\
\hline & Netherlands & $9 \%$ & $-9 \%$ & $-6 \%$ & 18 \\
\hline & Estonia & $9 \%$ & $-6 \%$ & $7 \%$ & 18 \\
\hline & Austria & $36 \%$ & $2 \%$ & $-7 \%$ & 17 \\
\hline & France & $-4 \%$ & $0 \%$ & $-1 \%$ & 17 \\
\hline & Slovakia & $18 \%$ & $27 \%$ & $35 \%$ & 16 \\
\hline & Sweden & $-15 \%$ & $-11 \%$ & $-7 \%$ & 15 \\
\hline & Latvia & $-19 \%$ & $13 \%$ & $8 \%$ & 15 \\
\hline & Czechia & $20 \%$ & $21 \%$ & $34 \%$ & 14 \\
\hline & Croatia & $-11 \%$ & $21 \%$ & $13 \%$ & 12 \\
\hline & Finland & $-21 \%$ & $-11 \%$ & $-19 \%$ & 9 \\
\hline & Bulgaria & $28 \%$ & $14 \%$ & $19 \%$ & 8 \\
\hline
\end{tabular}

Table A1: Mortality growth (expressed in \%) in different countries from Jan-May 2020 to Jan-May 2021 in different age groups. The variation in \% of the mortality rate, which was computed as the ratio of deaths per population in each age category variation and each age group, was compiled between the same periods of 2020 and 2021, from Jan 1st to end of May. Data was computed using various sources: (i) the mortality data in Israel was extracted from the Central Bureau of Statistics website, and deaths from the day of the Meron tragedy (30.04.2021) removed; (ii) the mortality data in England \& Wales was extracted from the Office of National Statistics; (iii) the mortality data in European countries was extracted from the Eurostat mortality database in those countries of above 1 million inhabitants (in 2020) with availability of the data for at least 20 weeks in 2021; (iv) and vaccination data which is extracted from Our World in Data. Sources: CBS, Eurostat, $\underline{\text { ONS. }}$

\begin{tabular}{|c|c|c|c|}
\hline & 20-29 & 30-39 & 40-49 \\
\hline Average mass vaccination countries (Israel, UK, Hungary) & $23 \%$ & $15 \%$ & $20 \%$ \\
\hline Average moderate vaccination countries ( 23 European countries) & $0 \%$ & $4 \%$ & $4 \%$ \\
\hline Difference mass - moderate & $22 \%$ & $11 \%$ & $16 \%$ \\
\hline p-value difference t-test & $3 \%$ & $11 \%$ & $6 \%$ \\
\hline
\end{tabular}

Table A2: Average 2021 to 2020 mortality growth (in \%) in mass and moderate vaccination countries for each age group (from Table A1). The p-value of a one-sided (equal variance) t-test was assessed on the difference in $\%$ of mortality between countries with mass vaccination and moderate vaccination. 